\title{
Comparison of Ramsauer and Optical Model Neutron Angular Distributions
}

D. P. McNabb, J. D. Anderson, R. W. Bauer, F. S. Dietrich, S. M. Grimes, C. A. Hagmann

October 4, 2004

International Conference on Nuclear Data for Science and Technology (ND2004) Santa Fe, NM, United States September 26, 2004 through October 1, 2004 
This document was prepared as an account of work sponsored by an agency of the United States Government. Neither the United States Government nor the University of California nor any of their employees, makes any warranty, express or implied, or assumes any legal liability or responsibility for the accuracy, completeness, or usefulness of any information, apparatus, product, or process disclosed, or represents that its use would not infringe privately owned rights. Reference herein to any specific commercial product, process, or service by trade name, trademark, manufacturer, or otherwise, does not necessarily constitute or imply its endorsement, recommendation, or favoring by the United States Government or the University of California. The views and opinions of authors expressed herein do not necessarily state or reflect those of the United States Government or the University of California, and shall not be used for advertising or product endorsement purposes. 


\title{
Comparison of Ramsauer and Optical Model Neutron Angular Distributions
}

\author{
D. P. McNabb, ${ }^{1}$ J. D. Anderson, ${ }^{1}$ R. W. Bauer, ${ }^{1}$ F. S. Dietrich, ${ }^{1}$ S. M. Grimes, ${ }^{2}$ \\ C. A. Hagmann ${ }^{1}$ \\ ${ }^{1}$ Lawrence Livermore National Laboratory \\ ${ }^{2}$ Ohio University
}

\begin{abstract}
The nuclear Ramsauer model is a semi-classical, analytic approximation to nucleon-nucleus scattering that reproduces total cross section data at the $1 \%$ level for $\mathrm{A}>40, \mathrm{E}_{\mathrm{n}}=5-60 \mathrm{MeV}$ with 7-10 parameters. A quick overview of the model is given, demonstrating the model's utility in nuclear data evaluation. The Ramsauer model predictions for reaction cross section, elastic cross section, and elastic scattering angular distributions are considered. In a recent paper it has been shown that the nuclear Ramsauer model does not do well in predicting details of the angular distribution of neutron elastic scattering for incident energies of less than $60 \mathrm{MeV}$ for ${ }^{208} \mathrm{~Pb}$. However, in this contribution it is demonstrated that the default angular bin dispersion most widely used in Monte Carlo transport codes is such that the observed differences in angular shapes are on too fine a scale to affect transport calculations. Simple studies indicate that 512-2048 bins are necessary to achieve the dispersion required for calculations to be sensitive to the observed discrepancies in angular distributions.
\end{abstract}

\section{INTRODUCTION}

The nuclear Ramsauer model has been used for decades to explain features of the neutron total cross section results. ${ }^{1,2}$ When precision data ${ }^{3}$ (with typical errors of only a few percent) became available it was quite surprising that such a simple model could adequately represent these data. ${ }^{4}$ A theoretical investigation ${ }^{5}$ of this "single-phase-shift slug model" demonstrated that nuclear refraction essentially made all neutron path lengths in the nucleus equal for neutron energies from a few $\mathrm{MeV}$ to approximately 60 $\mathrm{MeV}$. Franco ${ }^{6}$ had previously demonstrated that one did not need a "single phase shift" but merely that the average of the phase shifts varied smoothly with energy. Subsequently a large amount of precision neutron total cross section data $^{7}$ was successfully fit with this model. ${ }^{8}$

Azam and Gowda ${ }^{9}$ have recently proposed a more stringent test of the Ramsauer model and have compared calculated angular distributions of elastically scattered neutrons with optical model calculations. The shape of the angular distribution derived from the Ramsauer model did not agree in detail with the angular distributions derived from the optical model except for the forward maximum.

The point of this article is to demonstrate that in spite of deficiencies in calculating angular distributions with the Ramsauer model, it may be adequate for Monte Carlo neutron transport calculations $^{10,11,12}$ and may give more accurate results than using an optical model if these optical model parameters have not been carefully fit to new, high precision neutron total cross section measurements. The Ramsauer model also has several practical features that make it generally useful: (1) it requires only 5-10 parameters to globally represent the total neutron cross section within $2 \%$ for nuclei between calcium and uranium over the energy range of 5 to 60 $\mathrm{MeV}$, (2) it can be computed algebraically, and (3) it gives a reasonably accurate intuitive picture of the correct scaling relationships for baryon number, isospin, and incident neutron energy. One example demonstrating how the Ramsauer model can be used in nuclear data evaluation is given in Figure 1, where high-precision total cross section data ${ }^{7}$ for ${ }^{232} \mathrm{Th}$ and ${ }^{238} \mathrm{U}$ have been scaled using the Ramsauer model to 
generate a $1 \%$ estimate of the neutron total cross section for ${ }^{235} \mathrm{U}$ for $\mathrm{E}_{\mathrm{n}}>5 \mathrm{MeV}$.

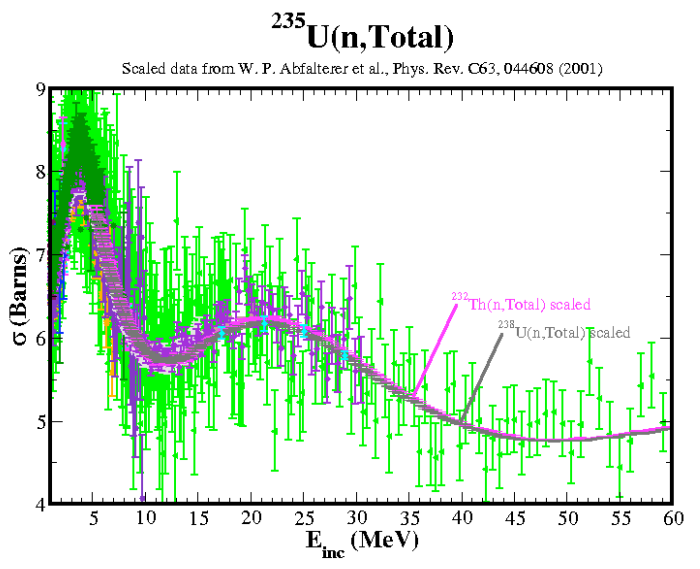

FIGURE 1. High-precision data from [7] has been scaled $\left({ }^{232} \mathrm{Th}\right.$ by $0.997-1.018,{ }^{238} \mathrm{U}$ by $\left.0.990-0.996\right)$ to estimate the ${ }^{235} \mathrm{U}$ (n,total) cross section to $1 \%$. Other data pictured was extracted from EXFOR. ${ }^{12}$

\section{RAMSAUER \& OPTICAL MODEL FITS}

For the purpose of neutron transport calculations we consider the optical model as merely a convenient way of representing a large quantity of measurements. The recent model calculations of Koning and Delaroche ${ }^{13}(K \& D)$ are an excellent representation of the newer, more precise total cross section measurements. ${ }^{7}$ We therefore take the cross sections generated by $\mathrm{K} \& \mathrm{D}$ as our standard for comparison.

Different fits to the total cross section data for $\mathrm{n}+$ ${ }^{208} \mathrm{~Pb}$ are plotted in Fig. 2. The K\&D optical model fits (circa 2003) are compared with fits by Becchetti and Greenlees ${ }^{14}$ (circa 1969) and with the Ramsauer model fit results from Ref. 8. Inspection of Fig. 2 indicates that for $\mathrm{n}+{ }^{208} \mathrm{~Pb}$ the Ramsauer model fits are significantly better at representing the total cross section data than the $B \& G$ optical model fit. In fairness, however, it should be noted that the Becchetti and Greenlees $(B \& G)$ fits did not have access to newer data that indicates that the imaginary potential decreases at lower neutron energies.

While the Ramsauer fits did not take into account elastic or reaction cross section data, they do a reasonable job of predicting these quantities. For neutron energies $10<\mathrm{En}<40 \mathrm{MeV}$, the Ramsauer model is arguably better than the $B \& G$ fit at representing the elastic and reaction cross sections.

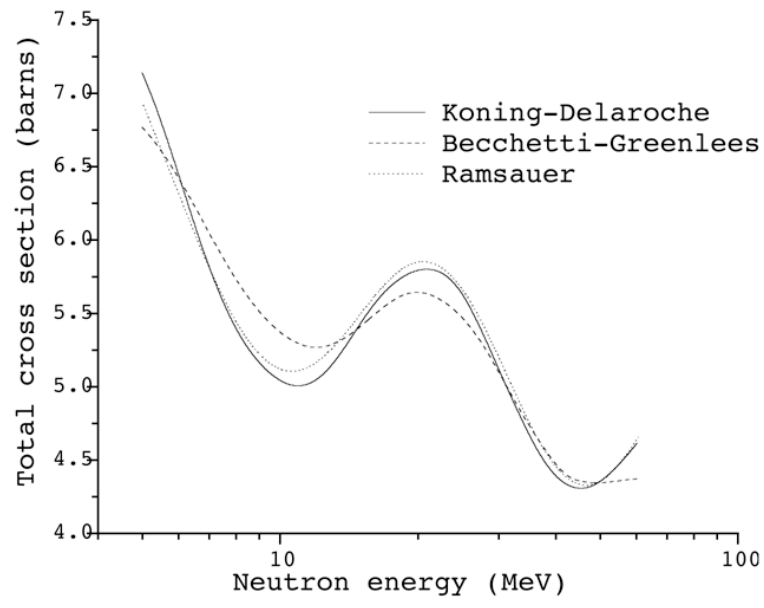

FIGURE 2. Different model fits to ${ }^{208} \mathrm{~Pb}$ (n,total) data.

\section{RAMSAUER DIFFERENTIAL ELASTIC SCATTERING DISTRIBUTIONS}

The Ramsauer model also reproduces the angular distribution of elastically scattered neutrons near zero degrees as shown at $E_{n}=15.45 \mathrm{MeV}$ in Fig. 3. Plotted in Fig. 4 is what Azam and Gouda called the "relative differential shape-elastic cross section" $\square$ defined as $\square \equiv \frac{\square_{e l}(\square)}{\square_{e l}}=\frac{1}{4 \square}\left[\begin{array}{l}\square \\ \square\end{array} \quad(2 l+1) P_{l}(\cos \square)\right.$

The quantity $\square$ is typically tabulated and sampled in Monte Carlo transport codes when sourcing elastically scattered neutrons to determine the scattering angle. The energy of the elastically scattered neutron is then determined using two-body kinematics.

The Ramsauer model compares favorably with the optical model calculations for the forward maximum (0-20 degrees) in the elastic scattering distribution. The average elastic cross section is also well described (to a few percent) by the Ramsauer model. However, beyond 30 degrees Azam and Gouda ${ }^{9}$ have demonstrated large discrepancies between angular distributions as calculated with the Ramsauer and optical models (see Figs. 12 through 21 in Ref. 9).

Three features of optical model calculations that lead to less deep minima in the angular distributions than are present in the Ramsauer model are (1) the use of distorted waves instead of plane waves, (2) an imaginary potential which has a different form factor from the real potential, and (3) the spin-orbit interaction which leads to different form factors for neutron parallel and anti-parallel couplings of the 
neutron spin and orbital angular momentum vectors. Of these three effects, the correct use of distorted waves plays the most significant role in removing the deep minima and in making the diffraction pattern more irregular. One simple extension to the Ramsauer model that removes the deep minima is to account for the above-mentioned effects by introducing a second term with a different radius that adds incoherently to the first; perhaps analogous to the spin-orbit potential which leads to two incoherent terms in optical model potentials. However, we believe the complications of this extension outweigh its benefits.

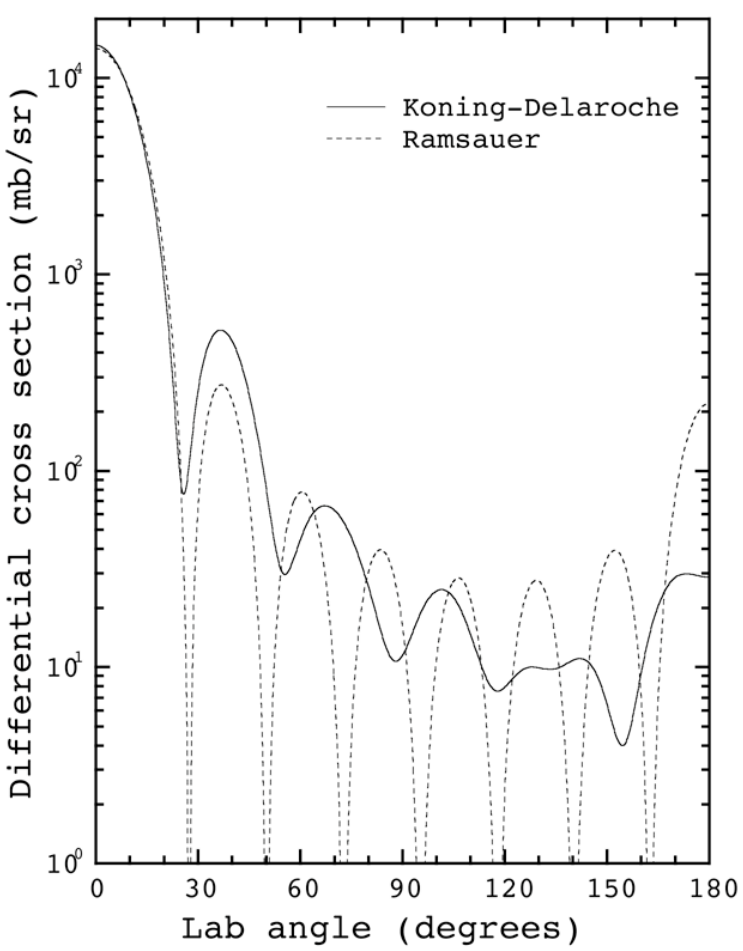

FIGURE 3. Angular distributions of elastically scattered neutrons for $E_{n}=15.45 \mathrm{MeV}$. The Koning and Delaroche optical model is compared with the Ramsauer model for ${ }^{208} \mathrm{~Pb}$.

From the perspective of neutron transport, smallangle elastic scattering and average elastic scattering properties are well described by the Ramsauer model for $5<\mathrm{E}_{\mathrm{n}}<60 \mathrm{MeV}$. Next we show that the average large-angle scattering, which results in neutron removal from the beam, is well described.

\section{NEUTRON TRANSPORT}

Most neutron Monte Carlo codes (e.g. MCNP ${ }^{10}$, $\mathrm{TART}^{11}$ ) use the "equally-probable-bin" method for sampling scattering angles. The angular distribution tables are a list of $\square=\cos \square$ values representing histogram boundaries. The default data tables for these codes have 32 cosine bins, tabulated at a number of incident neutron energies.

The "equally-probable-bin" method tends to smear out fine structure present in the original probability distribution. This smearing effect is markedly apparent in elastic neutron scattering distributions for $\mathrm{E}>5$ MeV. Fig. 4 shows calculated bin densities for the Ramsauer and the K\&D models when $\mathrm{E}=14 \mathrm{MeV}$ in ${ }^{208} \mathrm{~Pb}$. For 32 bins, the two models yield very similar bin boundaries. The fine details of the distributions become visible only by significantly increasing the number of "equally-probable-bins."

The angular distributions of both Ramsauer and optical models are dominated by a pronounced peak in the forward direction. We compared the predicted amounts of forward scattering by a running a Monte Carlo simulation and estimating the scattering cone size with respect to the forward direction. Fig. 5 shows the closely matching predictions of the K\&D and Ramsauer models as a function of energy.

We have performed several calculations to compare the effect of the angular distribution data on a simple penetration problem. The simplest of these is shown in Table 1 -- the transmissivity of an infinite slab for neutrons $\left(E_{n}=14 \mathrm{MeV}\right)$ at normal incidence is tabulated for slab thicknesses. Each value was obtained using the Monte Carlo method and averaging over $10^{7}$ histories. We have found that transport quantities have typically converged to $1 \%$ at 512 bins, though we occasionally found examples where convergence required 2048 bins. In all cases shown in Table 1, the Ramsauer model is preferable to an outof-date optical model fit to the data, in large part because the Ramsauer model is a better fit to highprecision cross section data.

\section{CONCLUSIONS}

For many applications of transport calculations, such as deep penetration through radiation shielding, the most important quantities are average elastic scattering properties, neutron removal and energy deposits. We have demonstrated that the Ramsauer model is reasonably accurate at describing these features. Given its simplicity, the Ramsauer model may be useful in fast neutron applications for calculating differential elastic scattering distributions 


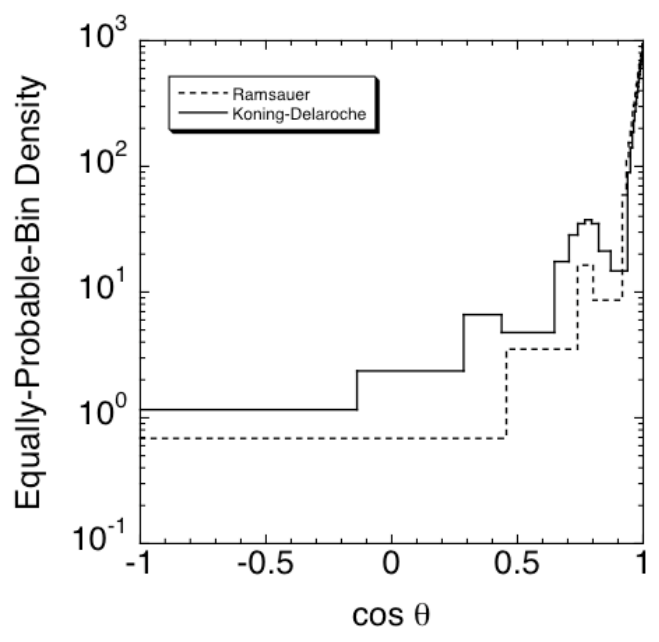

FIGURE 4. Equally-probable bin distributions for elastically scattered neutrons on ${ }^{208} \mathrm{~Pb}$ at $\mathrm{E}_{\mathrm{n}}=14 \mathrm{MeV}$.

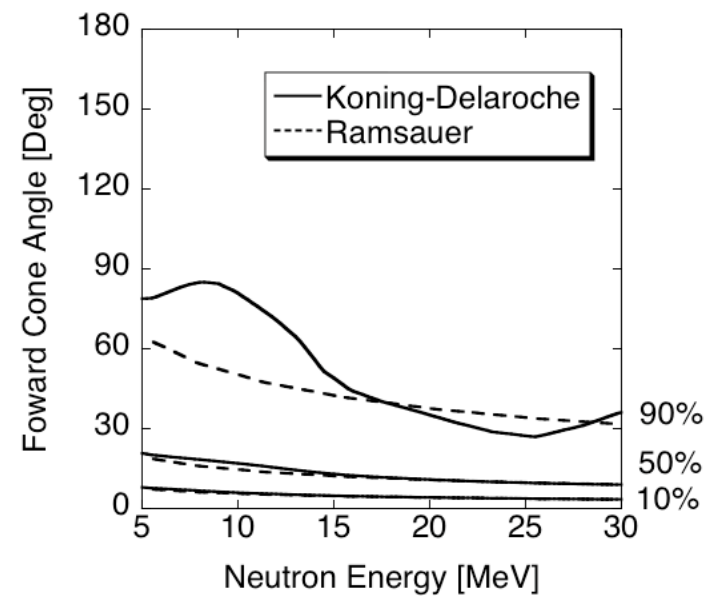

FIGURE 5. Elastic scattering forward cone opening angles for Ramsauer (dashed) and K\&D optical (solid) models based on 32 equally probable bins. The angles plotted are for $10 \%, 50 \%$, and $90 \%$ of the total neutron scattering probability of ${ }^{208} \mathrm{~Pb}$ at neutron energy $\mathrm{E}=14 \mathrm{MeV}$.

TABLE 1. Transmissivity through slab for $E_{n}=14 \mathrm{MeV}$ for several slab thicknesses given in units of number of ${ }^{208} \mathrm{~Pb}$ atoms per unit area.

\begin{tabular}{llll}
\hline & $\mathbf{1 0} / \mathbf{c m}^{\mathbf{2}}$ & $\mathbf{1 0} / \mathbf{c m}^{\mathbf{2}}$ & $\mathbf{1 0}^{\mathbf{2 4}} / \mathbf{c m}^{\mathbf{2}}$ \\
\hline Ram 32-bin & $0.9722(3)$ & $0.7524(2)$ & $0.04949(6)$ \\
Ram 2048-bin & $0.9724(3)$ & $0.7540(2)$ & $0.05011(6)$ \\
KD 32-bin & $0.9738(3)$ & $0.7613(2)$ & $0.04782(6)$ \\
KD 2048-bin & $0.9739(3)$ & $0.7622(2)$ & $0.04814(6)$ \\
BG 32-bin & $0.9716(3)$ & $0.7435(2)$ & $0.04080(6)$ \\
BG 2048-bin & $0.9713(3)$ & $0.7442(2)$ & $0.04115(6)$ \\
\hline
\end{tabular}

and reproducing the magnitude of integrated total, elastic, and reaction cross sections.

For applications where precision on the order of $1 \%$ or better is required, we have demonstrated that more careful attention must be paid to (1) accurately representing the relative magnitudes of the elastic and reaction cross sections and (2) improving the resolution of scattering angles over the current 32 equally-probable-bin method.

This work was performed under the auspices of the U.S. Department of Energy by the University of California, Lawrence Livermore National Laboratory under contract No. W-7405-Eng-48.

\section{REFERENCES}

1. J. D. Lawson, Phil. Mag., 44, 102 (1953).

2. J. M. Peterson, Phys. Rev., 125, 955 (1962).

3. H. S. Camarda, T. W. Phillips and R. M. White, Phys. Rev. C, 29, 2106 (1984).

4. J. D. Anderson and S. M. Grimes, Phys. Rev. C, $\underline{41}$, 2904 (1990).

5. S. M. Grimes, J. D. Anderson, R. W. Bauer and V. A. Madsen, Nucl. Sci. Eng., 130, 340 (1998).

6. V. Franco, Phys, Rev. B, 140, 1501 (1965).

7. R. W. Finlay, W. P. Abfalterer, G. Fink, E. Montei, T. Adami, P. W. Lisowski, G. L. Morgan, and R. C. Haight, Phys. Rev. C, 47, 237 (1993); W. P. Abfalterer, F. B. Bateman, F. S. Dietrich, R. W. Finlay, R. C. Haight, and G. L. Morgan, Phys. Rev. C, $\underline{63}, 044608$ (2001).

8. R. W. Bauer, J. D. Anderson, S. M. Grimes, D. A. Knapp and V. A. Madsen, Nucl. Sci. Eng., 130, 348 (1998).

9. M. Azam and R. G. Gowda, Nucl. Sci. Eng., 144, 86 (2003).

10. J. F. Briesmeister (Ed.), Los Alamos National Laboratory Report LA-12625-M, Los Alamos, NM (1997).

11. T. R. Wilcox and E. M. Lent, Lawrence Livermore National Laboratory Report M-221, Livermore, CA (1989).

12. EXFOR Systems Manual, Brookhaven National Laboratory, BNL-NCS-63330 (1999).

13. A. J. Koning and J.-P. Delaroche, Nucl. Phys. A, $\underline{713}$, $231(2003)$

14. F. D. Becchetti and G. W. Greenlees, Phys. Rev., 182, 1190 (1969). 